OPEN ACCESS

Edited by:

Sushil Kumar Mahata,

University of California,

San Diego, United States

Reviewed by:

Miles Douglas Thompson, Rady Children's Hospital-San Diego, United States

Adolfo Rivero-Muller,

Turku Centre for

Biotechnology, Finland

*Correspondence:

Madhu Khullar

madhu.khullar@gmail.com

Specialty section:

This article was submitted to Cellular Endocrinology,

a section of the journal

Frontiers in Endocrinology

Received: 31 January 2017 Accepted: 29 August 2017 Published: 29 September 2017

Citation:

Khullar M, Cheema BS and Raut SK

(2017) Emerging Evidence of

Epigenetic Modifications in Vascular

Complication of Diabetes.

Front. Endocrinol. 8:237.

doi: 10.3389/fendo.2017.00237

\section{Emerging Evidence of Epigenetic Modifications in Vascular Complication of Diabetes}

\author{
Madhu Khullar ${ }^{1 *}$, Balneek Singh Cheema ${ }^{2}$ and Satish K. Raut ${ }^{1}$ \\ ${ }^{1}$ Department of Experimental Medicine and Biotechnology, Postgraduate Institute of Medical Education and Research, \\ Chandigarh, India, ${ }^{2}$ PAREXEL International, Chandigarh, India
}

Genes, dietary, and lifestyle factors have been shown to be important in the pathophysiology of diabetes and associated microvascular complications. Epigenetic modifications, such as DNA methylation, histone acetylation, and post-transcriptional RNA regulation, are being increasingly recognized as important mediators of the complex interplay between genes and the environment. Recent studies suggest that diabetes-induced dysregulation of epigenetic mechanisms resulting in altered gene expression in target cells can lead to diabetes-associated complications, such as diabetic cardiomyopathy, diabetic nephropathy, retinopathy, and so on, which are the major contributors to diabetes-associated morbidity and mortality. Thus, knowledge of dysregulated epigenetic pathways involved in diabetes can provide much needed new drug targets for these diseases. In this review, we constructed our search strategy to highlight the role of DNA methylation, modifications of histones and role of non-coding RNAs (microRNAs and long non-coding RNAs) in vascular complications of diabetes, including cardiomyopathy, nephropathy, and retinopathy.

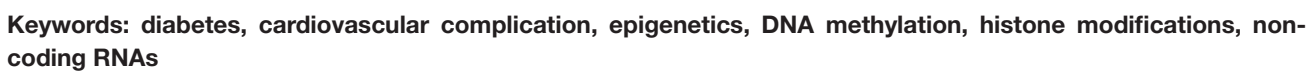

\section{INTRODUCTION}

In spite of adequate glycemic control, incidence of vascular complications associated with diabetes, such as diabetic cardiomyopathy, retinopathy, nephropathy, and neuropathy, remains high contributing to increased morbidity and mortality in diabetic patients. Recent studies suggest that a complex interplay between genes and environment may significantly contribute to pathogenesis of microvascular complications associated with diabetes (1-3). Emerging evidence suggests that environmental factors modulate aberrant expression of several key genes through epigenetic mechanisms in type II diabetes mellitus (T2DM) (4). Epigenetic changes, such as DNA methylation, histone modifications, and interference of RNAs, comprise the major epigenetic regulators of gene expression. A large volume of data has emerged supporting aberrant DNA methylation, histone modifications, and expression of microRNAs and long non-coding RNAs (lncRNAs) contributing to deregulation of signaling pathways (oxidative stress, inflammation, and apoptosis, etc.) in T2DM. However, our knowledge on epigenetic regulation in diabetes-associated microvascular complications remains limited. Thus, elucidation of epigenetic changes could provide better understanding of pathophysiology and therapeutic management of these diseases. In this article, we briefly summarize recent findings on the role of DNA methylation, histone modifications, and post-transcriptional RNA regulation in microvascular complications of diabetes. 


\section{SEARCH METHODOLOGY}

Literature searches of several electronic databases including Embase, Google Scholar, Ovid SP, Pubmed/Medline, and Web of Science were searched using the following search terms (free text, truncation, and MeSH or EMTREE terms): "DNA methylation" OR "histone acetylation," non-coding RNAs, microRNAs, long non-coding RNAs, post-transcriptional RNA regulation, epigenetic modifications, vascular, cardiovascular, renal, and retinal complications of diabetes for relevant publications in English language from 2006 to till date to evaluate the association between the role of DNA methylation, post-transcriptional RNA regulation, and histones modifications in diabetes-associated microvascular complications. Reference lists of included studies were hand-searched to identify other potentially eligible studies. Three authors (Madhu Khullar, Satish K. Raut, and Balneek Singh Cheema) reviewed the titles and abstracts to identify potentially eligible papers. These papers were examined in full detail. Final decision regarding inclusion was resolved by discussion. A manual review has been used for related publications and references of retrieved articles. We included randomized or non-randomized controlled clinical trials with or without blinding as well as cross-sectional and interventional studies that provided sufficient information.

\section{EPIGENETIC MODIFICATIONS IN DIABETES-INDUCED MICROVASCULAR COMPLICATIONS}

Evidence from both animal studies and clinical studies in diabetic patients has provided strong evidence linking histone modifications, post-transcriptional RNA regulation, and DNA methylation in microvascular complications of diabetes by regulating molecular pathways involved in pathophysiology of microvascular complications in diabetes (Figure 1).

These changes are inheritable and persist even after adequate glycemic control and contribute to metabolic memory and have

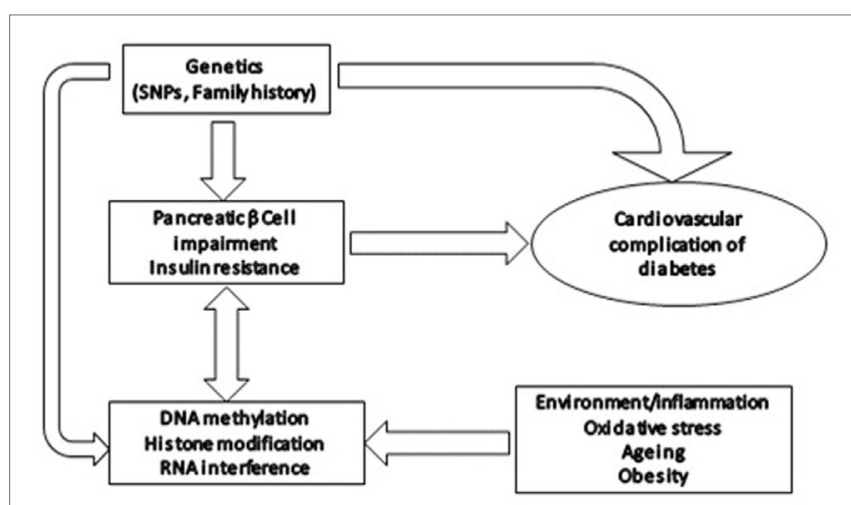

FIGURE 1 | Epigenetic modifications in diabetes: effect of various environmental/physiological factors on gene expression through epigenetic modifications, such as altered DNA methylation, histone modifications, and post-transcriptional RNA regulation. been suggested to significantly contribute to diabetes-induced vascular complications (5).

\section{HISTONE MODIFICATIONS IN DIABETES-INDUCED MICROVASCULAR COMPLICATIONS}

Histone acetyl transferases (HATs) and histone deacetylases (HDACs) are the enzymes involved in histone acetylation/ deacetylation and have been recently shown to be involved in regulating gene expression of several key molecules involved in microvascular complication of diabetes (6).

\section{HDACs IN DIABETES-INDUCED MICROVASCULAR COMPLICATIONS}

Histone deacetylases silence gene expression by deacetylating histone tails resulting in condensed euchromatin. Recent studies have implicated HDACs in diabetes and its associated microvascular complications; for example, HDAC1 and HDAC2 were shown to modulate expression of cardiac hypertrophy genes (6).

$\mathrm{O}$-linked $\beta$-N-acetylglucosamine (O-GlcNAc) is an important signaling molecule which regulates cell function through O-GlcNAcylation of serine and threonine residues of proteins (7). O-GlcNAc plays a central role in regulating cardiovascular function. Increased O-GlcNAc levels observed in diabetic hearts and have been linked to diabetic cardiomyopathy (8). Cox and Marsh have reported decreased levels of Mammalian switchindependent $3 \mathrm{~A}(\mathrm{mSin} 3 \mathrm{~A}), \mathrm{HDAC1}, \mathrm{HDAC2}$, and increased expression of HDAC2 mRNA and HDAC1/2 deacetylase activity in hearts from diabetic rats. These authors have suggested that there is a decreased physical association of O-GlcNAc with $\mathrm{mSin} 3 \mathrm{~A} / \mathrm{HDAC} 1 / 2$ in the heart which results in their altered activity and expression in the diabetic heart and impacts its function. However, physical exercise increased cardiac O-GlcNAc of these proteins resulting in beneficial effects on cardiac function and proposed that anti-hypertrophic effects of exercise on diabetic hearts were mediated by O-GlcNAc mediated post translation modification of HDAC1, 2 and $\mathrm{mSin} 3 \mathrm{~A}$ (9).

HDAC3 has been shown to exert pro-hypertrophic effect in diabetic mice. $\mathrm{Xu}$ et al. have reported significantly increased cardiac HDAC3 activity in the OVE26 diabetic mice. They showed that HDAC3 was exerting its pro-hypertrophic activity by downregulating DUSP5 (a MAP Kinase phosphatase) expression, by deacetylation of histone $\mathrm{H} 3$ in the primer region of DUSP5 gene (10). There are several studies showing beneficial and preventive effects of HDAC inhibition on diabetes-induced cardiovascular function. (This has been given in detail in a separate section.) However, further research is warranted to identify the specific HDAC isoforms that are dysregulated and their molecular targets that result in diabetic cardiomyopathy.

The role of HDACs in diabetic nephropathy has been reviewed recently by $\mathrm{Li}$ et al. (11). The available literature suggests that different HDAC isoforms targeting different molecular pathways are involved in pathophysiology of diabetic nephropathy. For example, HDAC1, HDAC2, and HDAC5 were shown to 
modulate expression of genes induced by TGF- $\beta 1$ (12), TGF- $\beta$ (13), and HDAC4 inhibited autophagy by deacetylating STAT1 (14).

Increased histone acetylation has been also reported in diabetic retinopathy and has been partly attributed to high glucosemediated decreased HDAC activity in retinal cells. HDAC activators and HDAC inhibitors were found to mitigate or potentiate diabetes-induced histone acetylation and expression of proinflammatory proteins in high glucose-treated cultured retinal Müller glia cells, confirming contribution of histone acetylation of retinal cells in pathophysiology of diabetic retinopathy (14). Decreased IL-10 levels are seen in diabetic retinopathy patients that have been suggested to be due to increased HDAC11 activity in conjunction with miR-19a in peripheral B cells of diabetic retinopathy patients (15).

Thus, available evidence supports a pathogenic role for aberrant HDAC activity in DC, DN, and DR by promoting histone acetylation and repression of genes of various signaling pathways, such as pro-inflammatory, pro-fibrotic, and antioxidant pathways.

\section{HATS IN DIABETES-INDUCED MICROVASCULAR COMPLICATIONS}

Histone acetylation mediated by HATs is another important epigenetic mechanism in gene regulation. HATs acetylate specific lysine residues of core histones at the $\mathrm{N}$-terminal tail, causing DNA uncoiling, increased accessibility to transcription factors, and increased gene expression. Thus, altered HAT activity could regulate gene expression and affect cell function. Indeed, HATs have been implicated in several diseases, such as cancer, diabetes, cardiac hypertrophy, asthma, and so on.

Recent evidence suggests that HATs may participate in the pathophysiology of microvascular complications of diabetes by regulating the expression of inflammatory pathway genes. For example, high glucose treatment of monocytes was found to increase transcriptional activity of HATs CBP and p/CAF, resulting in increased histone lysine acetylation of promoter regions of inflammatory genes, cyclooxygenase-2 (COX-2) and TNF- $\alpha$ gene, and increased gene expression of these cytokines in cultured monocytes (16). An increased promoter histone lysine acetylation of inflammatory genes has been reported in monocytes from both T1DM and T2DM patients (17). Furthermore, HAT-mediated lipid oxidation has been also found to increase inflammation by increasing histone acetylation of inflammatory genes (18). Yun et al. observed that HATs-mediated increased pro-inflammatory cytokine expression could be attenuated by curcumin in high glucose-treated human monocytes (19). Curcumin was shown to decrease high glucose-induced HAT activity, p300 gene expression, and acetylation of $C B P / p 300$, a complex that functions as a coactivator of NF- $\kappa B$. The role of HATs in diabetic nephropathy has been recently reviewed by Li et al. (11) and provides evidence that high glucose-induced increased activity and levels of HATs, such as $\mathrm{p} 300, \mathrm{CBP}$, and $\mathrm{p} / \mathrm{CAF}$, are mediating the activation of proinflammatory cytokines, ECM proteins, endothelial function, and fibrotic processes in diabetic nephropathy, via acetylation of both histone and non-histone proteins, such as Smads, p53, SP1, and NF- $\mathrm{KB}$.

\section{SIRTUINS IN DIABETES-INDUCED MICROVASCULAR COMPLICATIONS}

Recently, another class of HDACs, Sirtuins has been shown to regulate key cellular and metabolic processes by deacetylating the lysine residues of proteins involved in these processes. Sirtuins are a highly conserved protein family of HDACs and have been found to have protective effects against several diseases, such as diabetes, cancer, cardiovascular, and neurodegenerative diseases (20). Sirtuins exert these beneficial effects by modulating the expression of the genes involved in energy metabolism, DNA repair, inflammation, fibrosis, and oxidative stress (21).

Sirtuins regulate enzymes of carbohydrate metabolism, lipid metabolism, adipogenesis, and insulin secretion in diabetic patients (15). SIRT1 regulates glucose metabolism in liver, pancreas, muscle, and adipose tissue, mainly by regulating PGC- $1 \alpha$ (22). SIRT1 induces gluconeogenic genes through deacetylation of PGC-1 $\alpha$ in fasting state. FOXO group of transcriptional factors promote gluconeogenesis via STAT3; SIRT1 inhibits gluconeogenesis by inhibiting gluconeogenic genes via deacetylation of FOXO transcription factors and STAT3 in liver (22). Increased SIRT1 is also shown to increase glucose-induced insulin secretion in pancreatic $\beta$-cells which is partly due to SIRT1-mediated inhibition of UCP-2 in pancreatic islet $\beta$-cells (22). SIRT3, a mitochondrial protein deacetylase was found to be effective in increasing insulin sensitivity and decreasing serum glucose (16). SIRT4, another sirtuin involved in glucose homeostasis acts by repressing enzyme glutamate dehydrogenase (GDH) inhibiting insulin secretion (23). Decreased levels of SIRT1, 3, and 4 have been observed in diabetic patients and were associated with hepatosteatosis. Apart from this, sirtuins have been also shown to regulate activity of NFkB and expression of its downstream inflammatory genes in diabetes $(18,19)$.

Recent studies show that cardiac Sirtuins expression is dysregulated in diabetic patients. Bagul et al. reported a decrease in cardiac SIRT- 1 and increase in SIRT-3 activity in the T2DM rat and downregulation of all sirtuins except SIRT-2, which was increased in T1DM rat heart (24). In a recent review on sirtuins in cardiac complications of diabetes, sirtuins were suggested to attenuate the effects of insulin resistance and oxidative stress pathways in heart (25). SIRT-1 has been found to be the most important modulator of vascular function and is being targeted for therapeutic potential in various pre-clinical studies to improve cardiovascular functions. Bagul et al. have recently shown beneficial effect of reservatol on diabetic rat heart through modulating expression of SIRT-1 in T2DM and SIRT-1, 2, 3, and 5 in T2DM (24).

Recently, role of Sirtuins in vascular homeostasis has been reviewed nicely (26). Sirtuins were shown to regulate endothelial damage and vascular repair mechanisms. Sirtuins, by acting on specific endothelial targets, regulate several processes, including inflammation by modulating cytokine expression (IL-6, TNF$\alpha$, NF-KB, MMP-14), oxidative stress [manganese superoxide dismutase (MnSOD), FOXOs], and deacetylation of histone H3K14 and H4K16 (27).

High glucose milieu has been found to induce endothelial cell senescence and functional abnormalities by repressing 
SIRT1 expression high glucose-treated endothelial cells. SIRT1 upregulation in these cells was found to be protective against glucose-induced endothelial dysfunction indicating its potential protective role in diabetic vascular complications (21). Advanced glycation end products (AGEs), important mediators of diabetes, induced vascular abnormalities. AGEs have been shown to decrease SIRT1 levels and promote apoptosis in human endothelial Eahy926 cells which could be reversed by increasing SIRT1, confirming that AGEs were inducing apoptosis by repressing SIRT1 in endothelial cells (28). The downregulation of SIRT1 by high glucose and in diabetic has been proposed to be mediated by glucose-induced oxidative stress in endothelial cells. Mortuza et al. showed that high glucose-induced downregulation of SIRT1 was accompanied by FOXO1-mediated decreased levels of antioxidant enzyme, suggesting that SIRT1/FOXO1 axis was regulating oxidative status in endothelial cells (27).

Decreased SIRT1 expression has been also implicated in increased cellular senescence in renal glomerulus and retinal blood vessels in diabetic male C57BL/6 mice which were mediated by $\mathrm{p} 300$ and FOXO1 mediated reduction in mitochondrial antioxidant enzyme MnSOD in these cells (27). Furthermore, SIRT1 overexpression has been also shown to be protective in diabetes-induced renal and retinal injury in diabetic mice, through attenuated p300, endothelin-1 (ET-1), and TGF- $\beta 1$ expression (29). Downregulation of SIRT1 has been also shown to promote diabetic retinopathy by inducing increased MMP-9 expression in retinal endothelial cells (RECs) via acetylating transcriptional factor AP-1 (30). AGEs have been recently reported to decrease SIRT3 levels and SIRT3 knock down was associated with endothelial dysfunction in endothelial progenitor cells (EPCs). Moreover, SIRT3 augmentation ameliorated cellular dysfunction and enhanced antioxidant machinery (31). SIRT6 deficiency has been found to impair wound healing in diabetic $\mathrm{db} / \mathrm{db}$ mice and induce pro-inflammatory cytokines and oxidative stress, and decrease angiogenesis, suggesting its potential role in diabetic vasculopathy (32).

The role of other sirtuins in diabetic vascular complications is not known and needs to be investigated. Overall, diabetesinduced downregulation of sirtuins (SIRT1, 3 and 6) appears to promote oxidative stress and endothelial dysfunction, and induce cellular fibrosis, suggesting these molecules to be of potential therapeutic use in diabetes and associated vascular complications.

\section{HISTONE METHYLATION IN DIABETES-INDUCED MICROVASCULAR COMPLICATIONS}

Methylation of core histone tails at lysine or arginine residues are known to modulate gene expression by changing chromatin structure. For example, methylation at H3-K9 and H3-K27 mediates heterochromatin formation and results in silencing gene expression. Aberrant histone lysine methylation has been found to be involved in several pathological processes such as cancer, diabetes, cardiovascular diseases, etc. High glucose has been shown to induce increased histone $\mathrm{H} 3$ lysine 9 dimethylation in THP1 monocytes. Miao et al. showed that high glucose exposure caused increased $\mathrm{H} 3 \mathrm{~K} 4 \mathrm{me} 2$ and $\mathrm{H} 3 \mathrm{~K} 9 \mathrm{me} 2$ of specific chromatin regions and their associated genes. They reported increased $\mathrm{H} 3 \mathrm{~K} 4 \mathrm{me} 2$ was associated with increased methylation of nine genes, including ICAM3, FOS, GSTA-4, IL-8, and BCL-9, showed decreased methylation following HG exposure. Similarly, H3K9me2 methylation resulted in increased methylation of 39 genes and decreased methylation of 11 genes. They further observed increased $\mathrm{H} 3 \mathrm{~K} 9 \mathrm{me} 2$ at the coding and promoter regions of two candidate genes (IL-1A and PTEN) in blood monocytes of diabetic patients, indicating that diabetic milieu induced aberrant histone methylation is an important contributor to diabetesassociated complications (33).

Histone methyl transferases (HMTs) carry out methylation at specific lysine or arginine residues. HMTs Suv39 and G9a family methylate histone $\mathrm{H} 3$ at Lys9 and cause gene silencing whereas SET1/2 family HMTs methylate histone H3 at Lys4 and correlate with gene activation. Okabe et al. reported sustained vascular gene expression of H3K4 methyl transferase, Set7 as a responsive measure to hyperglycemia in vascular endothelial cells. They showed that metabolic memory of prior exposure to hyperglycemia was induced by Set7 and proposed that Set7 was a potential molecule for the phenomenon of hyperglycemic memory (34). This was further supported by an another study which showed that high glucose exposure altered ratio of cytoplasmic/nuclear ratio of Set7 protein without changing overall level of Set7 in vascular endothelial cells, indicating a role of Set7 and its role in hyperglycemia-induced gene activation of vascular endothelial cells (35).

The role of histone methylation in diabetic retinopathy has been also documented. For example, Zhong et al. showed that retinal superoxide dismutase gene (SOD2) was epigenetically regulated in diabetes through methylation/acetylation of $\mathrm{H} 4 \mathrm{~K} 20 \mathrm{me}$, acetyl H3K9, and NF-kB p65 on the histones at the promoter/enhancer location of retinal SOD2 in diabetes (36). These authors showed that these modifications continued after termination of hyperglycemia, supporting a diabetes-induced epigenetic regulation of retinal SOD2 (36). Their study suggests that promoter region methylation of SOD2 histones might play an important role in progression of diabetic retinopathy. Similarly, H3K9-specific demethylase JHDM2A (also known as JHMJD1A and KDM3A) has also been shown to be involved in regulating the expression of metabolic genes, strengthening the role of epigenetic regulation of metabolic genes in microvascular complications of diabetes (37). These authors observed that JHDM2A regulates the expression of PPAR $\alpha$ and $\beta$-adrenergic signaling pathway genes and suggested that JHDM2A might regulate energy mediated $\beta$-adrenergic signaling pathway (37).

The fetal exposure to maternal milieu such as nutrition is known to result in intrauterine growth restriction (IUGR) and influence susceptibility to several diseases such as insulin resistance in adults. Hepatic insulin growth factor 1 (IGF-1) modulates insulin sensitivity, thus decreased IGF-1 levels are linked to insulin resistance. Decreased post natal plasma IGF-1 levels have been reported in IUGR infants and in new born rats with induced IGUR (38). Fu et al. have shown that IUGR affects IGF- 1 gene expression by modulating the region 
and gender-specific histone modifications (methylation and acetylation) along the length of IGF-1 gene. The authors showed that IUGR significantly increased $\mathrm{H} 3 \mathrm{~K} 4 \mathrm{me} 2$ in males and $\mathrm{H} 3$ K4me3 in females new born rats with induced IUGR. Since, there is a dynamic association between histone methylation and associated DNA methylation which affects gene transcription, these histone modifications resulted in decreased IGF-1 expression in new born rats. These findings suggest that that aberrant methylation of core histone tails of hepatic IGF-1 regulate IGF-1 expression.

Yu et al. (39) have shown that combination of diabetes and renal failure accelerated cardiomyopathy by epigenetic alterations (increased acetylation, phosphorylation, K4 dimethylation, and reduced K9 dimethylation) of the cardiac histones $\mathrm{H} 3$. They observed increased H3 dimethylation at lysine 4 and 9 and decreased $\mathrm{H} 3$ dimethylation at lysine 9 in hearts of uninephrectomized $\mathrm{db} / \mathrm{db}$ mice resulting in transcriptionally active chromatin and proposed that these changes were associated with increased expression of cardiac hypertrophy related genes. However, factors causing these changes are not known and need to be determined.

Histone methylation in etiology of diabetic nephropathy has been widely investigated and reviewed recently (40). Diabetic nephropathy is characterized by glomerular mesangial expansion, inflammation, renal fibrosis, and hypertrophy. In a recent study, Li et al. (41) showed that increased p21 expression seen in high glucose-treated mesangial cells was mediated by reduced histone H3-lysine9-dimethylation ( $\mathrm{H} 3 \mathrm{~K} 9 \mathrm{me} 2)$, increased histone $\mathrm{H} 3$-lysine 4 methylation $(\mathrm{H} 3 \mathrm{~K} 4 \mathrm{me} 1 / 3)$ and increased translocation of SET7/9 at the p21 promoter region. Similarly, Yuan et al. (12) also showed that oxidized lipid products such as 2(S)-hydroxyeicosatetraenoic acid [12(S)-HETE] increased transcriptional activity of SET7, which in turn increased expression of pro-fibrotic genes in HETE treated mesangial cells. Losartan, an AT1R inhibitor, a common drug used in treatment of diabetic nephropathy has been shown to decrease H3K9/14Ac at RAGE, PAI-1, and MCP-1 promoters, in mesangial cells from $\mathrm{db} / \mathrm{db}$ diabetic mice, suggesting that AT1R action may be also mediated by attenuation of epigenetic changes of the key genes involved in diabetic nephropathy.

Altered histone methylation of RECs has been reported in diabetic retinopathy. For example, decreased expression of MnSOD was found to be associated with altered H3K4me1/ me2in diabetic retinas and endothelial cells (42). These changes were found to persist even after normalization of blood glucose levels, indicating that these changes acted as markers of metabolic memory (42).

Decreased H3K9me2 promoter methylation of MMP9, promoting increased expression has been also seen in diabetic retinas and suggested to be associated with increased ECM accumulation (42). Increased expression of PRMT4, a methyltransferase which specifically methylates H3R17 histones and promotes cell death has been observed in retinal pigment epithelial layer of diabetic rats even before development of diabetic retinopathy (43). Wang et al. (44) reported differential methylation on $\mathrm{H} 3, \mathrm{H} 4, \mathrm{H} 2 \mathrm{~A}, \mathrm{H} 2 \mathrm{~B}$, and $\mathrm{H} 1$ sites in diabetic retinas specifically they observed increased mono- and dimethylation of histone $\mathrm{H} 4$ lysine 20 (H4K20me1/me2), and were associated with DNA damage in retinas of diabetic rats and these methylation patterns could be partly reversed by minocycline, a strong neuroprotective drug and used in treatment of diabetic retinopathy. Thus, altered histone methylation appears to be important in development of diabetic retinopathy in animal models and in vitro conditions, however, these changes need to be replicated in diabetic patients.

Thus, in summary, hyperglycemia-induced differential histone methylation/acetylation appears to regulate expression of several genes of cellular pathways, such as endothelial activation, oxidative stress, adrenergic signaling pathway, and so on, involved in diabetes-induced vascular complications.

\section{MODULATION OF HDACs AND HATS AS A THERAPEUTIC APPROACH}

Since HDACs along with HATs have been shown to have a critical role in regulating expression of genes involved in diabetic vascular complications, modulation of these molecules is being investigated for therapeutic applications in diabetic cardiomyopathy, nephropathy, retinopathy, and endothelial dysfunction associated with diabetes (45).

For example, acetylation of $20 \mathrm{~S}$ proteasome subunits in the heart has been shown to mediate proteolytic activity of injured myocardium (46), it has been suggested that modulation of HDACs, the key regulators of acetylation in the cell could be used effectively in the treatment of cardiac injury $(47,48)$. Christensen et al. reported that HDAC inhibition could ameliorate late diabetic microvascular complications along with improving insulin resistance and $\beta$-cell function (49). HDAC inhibition was also shown to improve cardiac function and attenuated cardiac remodeling in the diabetic myocardium of the streptozotocintreated ICR mice. Chen et al. observed that diabetic mice given $1 \%$ butyrate in drinking water resulted in HDAC inhibition in the diabetic myocardium, specifically myocardial HDAC4 was found to be significantly decreased. HDAC inhibition caused upregulation of GLUT 1 and 4, increased Caspase 3, increased myocardial superoxide dismutase, decreased cardiac interstitial fibrosis and myocyte hypertrohy resulting in improvement in cardiac performance in diabetic mice (50).

Chen et al. have also recently shown that HDAC inhibition promotes stem cell-derived myocardial repair, thereby improving cardiac function and attenuating cardiac remodeling in diabetic rats, further confirming a protective role of HDAC inhibitors against myocardial injury (50).

Peroxisome proliferator-activated receptors (PPARs) play an important role in diabetes-associated heart diseases by regulating cardiac glucose and lipid homeostasis. HDAC inhibitor, MPT0E014, was shown to decrease cardiac inflammation and dyslipidemia by modulating myocardial PPARs, and attenuated diabetic cardiomyopathy (51).

DUSP 5 is a dual-specific phosphatase which dephosphorylates and inactivates ERK1/2 MAP Kinase, a known promoter of cardiac hypertrophy (10). Xu et al. recently reported that HDAC3 inhibition with its selective inhibitor, RGFP966, 
increased the expression of MAP kinase phosphatase, DUSP 5 and prevented development of diabetic cardiomyopathy in Type 1 diabetes OVE26 mice, suggesting a therapeutic potential of HDAC3 inhibition in prevention of diabetic cardiomyopathy (10).

Histone deacetylase inhibitors have been also found to be effective in preventing diabetes-induced renal damage. Gilbert et al. (52) reported that HDAC inhibitor, Vorinostat, blunted renal damage in diabetic rats by reducing renal growth and glomerular hypertrophy via modulating renal EGFR expression. Vorinostat has been also shown to attenuate renal damage in strptozotocin-treated mice by decreasing eNOS expression and oxidative stress (53). Valproic acid (VPA), a known HDAC inhibitor also has been shown to ameliorate diabetes-induced renal injury by inhibiting renal fibrosis (54). Increased oxidative stress is an important contributor to diabetic nephropathy; Dong et al. recently showed that sodium butyrate inhibited HDAC activity and elevated the expression of NRF2 and its downstream targets heme oxygenase 1 and $\mathrm{NAD}(\mathrm{P}) \mathrm{H}$ dehydrogenase quinone 1 . Deletion of the NRF2 gene completely abolished sodium butyrate activation of NRF2 signaling and protection against diabetes-induced renal injury (55). Trichostatin A (TSA), an antifungal antibiotic has been shown to inhibit HDACs 1, 3, and 4. TSA suppresses redox signaling by decreasing NADPH Oxidase 4 (Nox4) expression by inhibiting p300-HAT-dependent pathway in endothelial cells (56). Cao et al. showed that TSA decreased transverse aortic constriction (TAC), induced cardiac hypertrophy and phenylephrine (PE) or ET-1, and induced cardiomyocyte hypertrophy by inhibiting autophagy, and suggested that TSA-mediated HDAC inhibition suppresses load- or agonist-induced autophagy in stressed myocardium (57).

Pancreatic duodenal homeobox 1 (PDX1) is a transcription factor associated with pancreatic $\beta$-cell function and survival. PDX1 deficiency results in defective B-cell function and diabetes. Park et al. observed that IUGR decreased fetal and postnatal PDX1 levels by histone modification of $P D X 1$ gene in primary islets. IUGR promoted deacetylation of histones $\mathrm{H} 3$ and $\mathrm{H} 4$ by recruiting HDAC1 and corepressor Sin3A; and histone 3 lysine 4 (H3K4) was demethylated and histone 3 lysine 9 (H3K9) was methylated, resulting in silencing of the PDX1. These authors suggested that IUGR-induced PDX1 gene silencing in the $\beta$ cell was linked with development of T2DM (58).

Johnson and Marsh recently reported that treatment of Type 2 diabetic $\mathrm{db} / \mathrm{db}$ mice with a chemotherapeutic class 1 HDAC inhibitor, romidepsin (FK228), at a low dose [(0.56 mg/kg twice a week) for 8 weeks], decreased blood glucose reduction independent of plasma insulin level. These authors have suggested that these anti-diabetic effects of romidepsin were mediated through HDAC2-mediated potentiation of intracellular insulin signaling (59).

Thus, available information till date suggests that HDAC inhibition has beneficial effects in ameliorating diabetic microvascular complications by targeting multiple dysregulated pathways. However, its translation into an effective therapy requires further studies such as evaluating association between HDACs and environmental and genetic factors.

\section{DNA METHYLATION IN DIABETES-INDUCED MICROVASCULAR COMPLICATIONS}

DNA methylation involves methylation at $5^{\prime}$ position of cytosine residues in $\mathrm{CpG}$ islands, mostly in the promoter regions and is carried out by DNA methyl transferases (DNMTs). Promoter DNA methylation is an important epigenetic mechanism regulating gene expression and is known to be affected in various diseases, including cardiovascular diseases and diabetes (60). Altered DNA methylation of inflammatory genes, glucose, and lipid metabolism genes, genes involved in oxidative stress, has been reported in diabetes (61).

DNA methylation in vascular complications of diabetes have been investigated and reviewed in a recent review (62). El-Osta (63) reported that short-term exposure of aortic endothelial cells to high glucose-induced promoter DNA methylation of $N F-k B$ p 65 subunit, an important mediator of cardiac fibrosis. These authors showed that DNA methylation was mediated by hyperglycemia-induced increased methylglyoxal generation. Pirola et al. (35) observed that hyperglycemia significantly affects human vascular chromatin resulting in differential methylation and acetylation pattern with the transcriptional upregulation of genes involved in metabolic and cardiovascular disease. A good correlation was seen between hyper-acetylation and DNA methylation and induction of genes in glucose-treated cells, and suggested that hyperglycemia-induced gene induction was mediated by distinct changes in methylation and acetylation pattern of the genes.

Distinct promoter methylation profiling has been reported in diabetic hearts too. Movassagh et al. (64) examined DNA methylation profiles in left ventricular tissues from patients with idiopathic and end stage heart failure and observed increased promoter methylation of 3 genes, PECAM1, ARHGAP24, and $A M O T L 2$, related to angiogenesis in cardiomyopathic hearts, suggesting a role of DNA methylation-induced altered gene expression in cardiomyopathy.

However, DNA methylation pattern seen in diabetic hearts is distinct from that seen in heart failure patients (9). A specific DNA methylation CpG site of $\beta$-myosin heavy chain $(\beta-M Y H 7)$ gene that was found to be extensively methylated in T2DM hearts as compared to controls and $3 \mathrm{CpG}$ sites of failing human hearts. Similar DNA methylation changes were also seen T1DM hearts and in steroid induced diabetic hearts (9), suggesting altered DNA methylation of specific $\mathrm{CpG}$ site of $\beta-M H C$ may be contributing to ventricular dysfunction seen in diabetic patients.

In a similar study, Mönkemann et al. (65) reported altered methylation status of P53-inducible $p 21$ WAF1/CIP1 promoter, resulting in activation of apoptotic pathway leading to cell death of cardiomyocytes and cardiomyopathy in diabetic rats. They proposed that oxidative stress was the major trigger contributing to de novo methylation of p53-inducible $p 21$ WAF1/CIP1 gene.

Diabetes-induced oxidative stress is an important mediator of diabetes-associated cardiovascular complications. Zhong et al. (66) recently reported significant hypomethylation of KEAP1 promoter in diabetic cardiomyopathy patients, with concomitant 
increase in KEAP1 protein levels in these patients. KEAP1 protein is known to bind to NF-E2-related factor 2 (NRF2), and promotes its degradation. NRF2 is known to activate several antioxidant enzymes. Studies have proposed that reduction of NRF2 antioxidant system in diabetic hearts may alter redox balance and contribute to increased oxidative stress in the heart of diabetic patients (67).

Vecellio et al. (68) have recently reported a decreased proliferation, differentiation potential, and premature cell death of cardiac mesenchymal stem cells in T2DM patients. Furthermore, they observed hypermethylation of promoter $\mathrm{CpG}$ islands of genes of cell cycle and DNA repair genes along with reduced acetylation of histone $\mathrm{H} 3$ lysine 9 (H3K9Ac) and lysine 14 (H3K14Ac) and increased trimethylation of H3K9Ac and lysine 27. They proposed that reduced HAT activity in diabetic hearts was responsible for increased DNA CpG methylation resulting in decreased cell differentiation and proliferation of cardiac mesenchymal stem cells in diabetic cardiomyopathy. However, these effects could be reversed by increasing HAT activity, suggesting a potential therapeutic application of epigenetic modulators in diabetes-associated cardiovascular complications.

Decreased promoter methylation of liver X receptor $\alpha(L X R a)$ (69) and $A T 1 b$ angiotensin (67) receptor gene leading to their increased expression has been observed in diabetic hearts. TNF- $\alpha$-mediated increased promoter methylation of sarcoplasmic reticulum Ca-ATPases (SERCA2a) resulting in decreased SERCA2a expression has been observed in high glucose-treated cardiomyocytes (70). These results suggest that diabetic milieu can cause increased or decreased methylation of different genes, resulting in their aberrant expression in heart.

Altered methylation of several genes dysregulated in diabetic nephropathy and diabetic retinopathy has been reported in diabetic patients and in vitro studies $(35,71,72)$. A distinct differential promoter DNA methylation pattern has been reported in diabetic nephropathy patients with end-stage renal disease as compared to those who do not progress to this stage (73), suggesting that diabetic environment results in distinct epigenetic changes in specific genes, which could be used as prognostic biomarkers. Similarly, in diabetic retinopathy, Agardh et al. (74) reported differential DNA methylation of nearly 233 unique genes, with genes from natural killer cell-mediated cytotoxicity pathway genes to be hypomethylated in proliferative diabetic retinopathy (PDR) and suggested that this distinct methylation pattern could be used as a prospective marker of PDR. Mishra and Kowluru (75) have shown that increased DNA methylation of mitochondrial DNA (mtDNA) causes decreased transcription of mtDNA, impairing mitochondrial functions and increasing apoptosis in diabetic retinopathy. A dynamic balance between methyl cytosine and hydroxyl methylation of MMP9 was found to be important in MMP9 expression and in maintaining mitochondrial integrity and function in RECs and in preventing diabetic retinopathy (76). Diabetes-induced oxidative stress appears to be a major trigger of these epigenetic changes.

Thus, there is substantial evidence to suggest that hyperglycemia causes aberrant methylation of regulatory regions of several distinct genes resulting in their dysregulated expression. These molecular changes appear to be important in the pathogenesis of diabetes-induced microvascular changes in heart, kidney, and retina of the diabetic patients. Furthermore, diet, exercise, environment, and genetic factors, which are important contributors to risk of diabetes, are also potent modulators of epigenetic changes. Hence, their role in inducing epigenetic changes in microvascular complications in diabetic milieu needs to be explored.

\section{NON-CODING RNAs AND DIABETES}

Non-coding RNAs are non-protein coding RNAs, and include microRNAs, long non-coding RNAs (LncRNAs), circular RNAs, etc. have been identified as important regulators of gene expression. These molecules have been shown to be important in developmental, physiological, and pathological processes. Dysregulated expression of microRNAs and long non-coding RNAs (lncRNAs) has been implicated in various diseases, including vascular complications of diabetes.

\section{MICRORNAS ASSOCIATED WITH DIABETES-INDUCED CARDIOMYOPATHY}

MicroRNAs are small non-coding RNAs which regulate gene expression by mRNA degradation or translational repression of mRNAs. The role of microRNAs has been widely studied in diabetes and its vascular complications and has been reviewed recently in several articles $(17,77,78)$. Dysregulated expression of several microRNAs has been reported in Diabetic cardiomyopathy, retinopathy, nephropathy, and neuropathy regulating genes involved in diabetes (Table 1). Most of these microRNAs are involved in fibrogenesis, hypertrophy, apoptosis, inflammation, angiogenesis, and ECM accumulation. These functions are mainly regulated by microRNAs by regulating the expression of target genes involved in these cellular processes.

Several microRNAs have been reported to contribute to pathophysiological processes of diabetic cardiomyopathy, such as myocardial fibrosis, cardiomyocyte hypertrophy, cardiomyocyte apoptosis, and mitochondrial dysfunction (Figure 2). For example, miR-30c, miR-133a, miR-150, and miR-373 were found to be downregulated and miR-451 was found to be upregulated in diabetes-induced cardiomyocyte hypertrophy (84). Whereas, in diabetes-induced cardiac fibrosis, the expression of miR-133a was found to be decreased and the expression of miR-21 was significantly increased (84). miR-34a, miR-1, miR-206, miR-195, and miR-30d have been implicated in diabetes-associated cardiac apoptosis and mitochondrial dysfunction (84). Raut et al. showed that the expression of putative target genes of miR-30c (CDC42 and PAK1) were increased in hearts of diabetic rats and in $\mathrm{HG}$ treated cardiomyocytes $(85,86)$. miR-30c overexpression attenuated hyperglycemia-induced cardiomyocyte hypertrophy, whereas miR-30c inhibition resulted in myocyte hypertrophy in high glucose-treated cardiomyocytes, suggesting antihypertrophic potential of $\mathrm{miR}-30 \mathrm{c}$ in diabetic cardiomyopathy (85). miR-200c has been found to be pro-hypertrophic and its expression was shown to be significantly increased in diabetic hearts and in high glucose-treated cardiomyocytes. It was found to induce diabetes-associated cardiac hypertrophy by down 
TABLE 1 | Dysregulated microRNAs in microvascular complications of diabetes.

\begin{tabular}{|c|c|c|c|}
\hline MicroRNAs & Targets & Functions & Reference \\
\hline \multicolumn{4}{|l|}{ Nephropathy } \\
\hline miR-192 & TGF- $\beta$ & ECM & (53) \\
\hline $\mathrm{miR}-200 \mathrm{~b} / \mathrm{c}$ & & Collagen, fibrosis & (46) \\
\hline miR-21 & PTEN & Renal cell hypertrophy and Fibrosis & (8) \\
\hline miR-195 & $\mathrm{Bcl}-2$ & Podocyte apoptosis & $(70)$ \\
\hline miR-377 & Fibronectin & Fibrosis & (79) \\
\hline miR-29 family & Collagen I, III, IV & Fibrosis & (53) \\
\hline miR-93 & VEGF-A & Glomerular function & (80) \\
\hline \multicolumn{4}{|l|}{ Retinopathy } \\
\hline miR-146, miR-155, miR-132, miR-21 (upregulated in retina) & $\mathrm{Nf}-\kappa \beta$ & Pro-apoptosis of retinal pericytes & (81) \\
\hline $\begin{array}{l}\text { miR-17-5p, miR-18a, miR-20a, miR-21, miR-31, miR-155 } \\
\text { (upregulated in retinal endothelial cells) }\end{array}$ & Vascular endothelial growth factor & Vascular permeability & (82) \\
\hline $\mathrm{miR}-200 \mathrm{~b}$ & VEGF-A & Vascular permeability & (83) \\
\hline
\end{tabular}

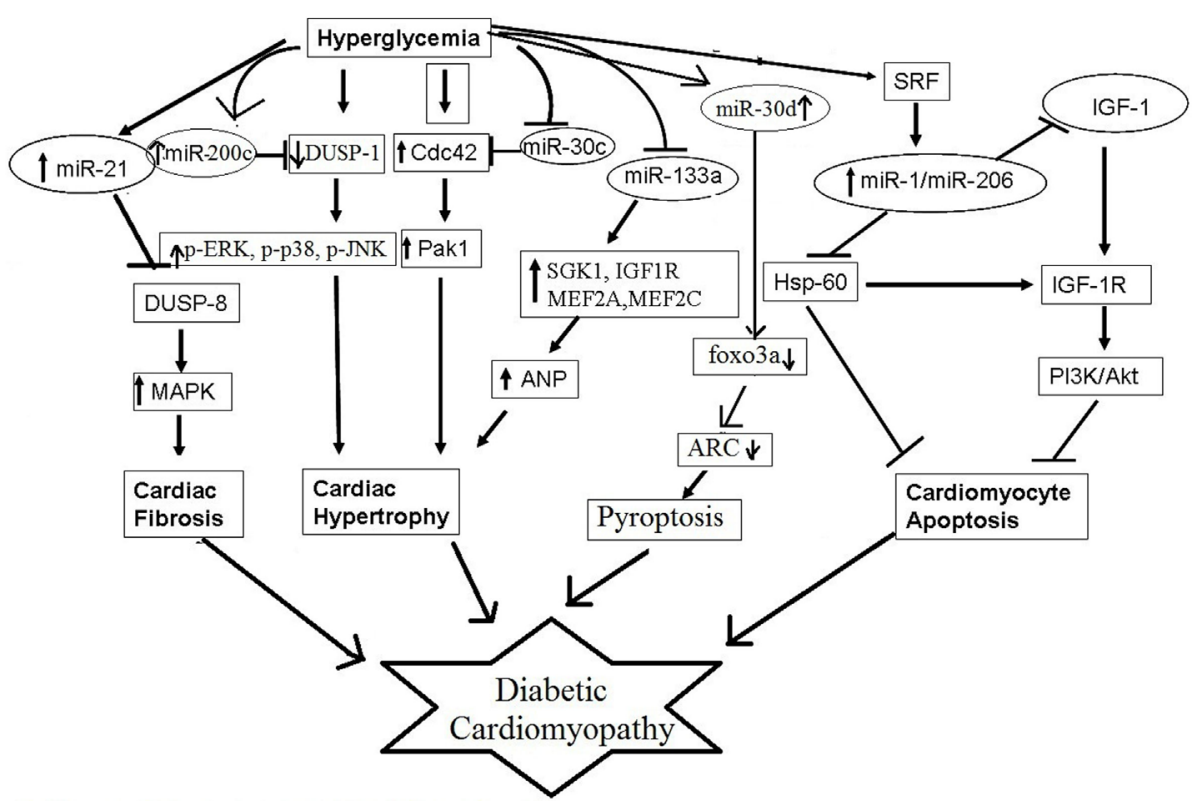

FIGURE 2 | Schematic model of epigenetic role of microRNAs in diabetic cardiomyopathy.

regulating expression of dual-specific phosphatase-1 (DUSP-1). Inhibition of miR-200c augmented the expression of the DUSP-1 causing decreased expression of phosphorylated ERK, p38, and JNK and attenuated cardiomyocyte hypertrophy in high glucosetreated neonatal rat cardiomyocytes (87). In another study, miR133 a expression was reduced in diabetic cardiomyopathy along with augmented gene expression of MEF2A, MEF2C, SGK1, and IGF1R. Over expression of this microRNA inhibited altered gene expression and hypertrophic changes, indicating that miR-133a participated in mediating glucose-induced cardiomyocyte hypertrophy in diabetes (88). Duan et al. (89) reported significantly reduced expression of miR-150 in high glucosetreated cardiomyocytes; this microRNA was shown to increase p300 expression, resulting in cardiomyocyte hypertrophy. miR-373 has also been shown to be involved in the pathogenesis of diabetes-induced cardiac hypertrophy. The expression of miR373 was found to be markedly down regulated in STZ-induced diabetic mice, and neonatal rat cardiomyocytes in response to high glucose. Over expression of miR-373 in cardiomyocytes using synthetic miR-373 mimics resulted in decreased expression of $M E F 2 C$ gene and attenuated cardiomyocyte hypertrophy in high glucose-treated cardiomyocytes (90). Kuwabara et al. identified calcium-binding protein 39 (CAB39), a component of AMPK signaling pathway as direct target of miR-451. They demonstrated that in miR-451 knockout mouse the protein expression of CAB39 and phosphorylated AMPK was increased significantly, indicating that $\mathrm{miR}-451$ was involved in diabetic cardiomyopathy via suppression of the LKB1/AMPK signaling pathway (91).

Several miRNAs, such as miR-21 and miR-29, have been shown to promote cardiac fibrosis in diabetic hearts. Liu et al. demonstrated increased miR-21 expression after high glucose treatment in cardiac fibroblasts (92). miR-21 was shown to promote fibroblast survival by down regulating SPRY1 (93). 
Silencing of miR-21 using synthetic mimics in mouse model of diabetic cardiomyopathy resulted in decreased interstitial fibrosis, suggesting potential role of miR-21 in cardiac fibrosis associated with diabetic cardiomyopathy. Liu et al. also showed that gain and loss of miR-21 function negatively regulated expression of DUSP8, a MAPK phosphatase, and enhanced cell proliferation and collagen synthesis via MAPK signaling pathway (92). Kumar et al. (94) have reported that miR-21 may also promote cardiac fibrosis by activation of $\mathrm{AKT} / \mathrm{PKB}$ signaling. In addition to cardiac hypertrophy, miR-133a was found to mediate diabetesinduced cardiac fibrosis. Chen et al. observed that miR-133a expression was significantly decreased in hearts of STZ-induced diabetic mice, along with increased expression of transcriptional co-activator p300, as well as fibrosis markers (95).

miR-1 and miR-206 are cardiac-specific microRNAs (96). Increased miR-1 and miR-206 levels have been observed in high glucose-treated cardiomyocytes. Both these miRNAs were proposed to induce cardiomyopathy by inducing mitochondrial dysfunction and apoptosis (97). These microRNAs have been shown to bind to the same site in the $3^{\prime}$-UTR of HSP60 mRNA and thereby could regulate HSP60 expression and glucosemediated apoptosis in diabetic myocardium; however, this needs experimental validation (97). miR-34 too has been shown to promote HG-induced apoptotic changes in H9C2 cells (96). Pyroptosis is pro-inflammatory programmed cell death and is unlike from apoptosis or necrosis (26). Li et al. in their study showed that miR-30d expression was substantially increased in diabetic cardiomyopathy and this increased expression promoted cardiomyocyte pyroptosis; conversely, knockdown of miR-30d attenuated it (98).

It has been proposed that diabetic milieu may induce or repress the microRNAs by several different mechanisms, such as oxidative stress and ER stress, epigenetically regulating genes coding for microRNAs.

Role of microRNAs in diabetic nephropathy has been investigated widely and there are several recent reviews on this topic (99). Existing literature supports a pathogenic role for several microRNAs by promoting renal fibrosis by increased accumulation of extracellular matrix proteins related to fibrosis, glomerular hypertrophy, and renal cell apoptosis (100). Some of these microRNAs have been shown to have a potential as biomarkers as these were found to be dysregulated in early stages of nephropathy. However, more evidence is required for these data to be translated to clinical application. Furthermore, it has been observed that modulation of these microRNAs with either mimics or antagomiRs could attenuate the disease, suggesting that these microRNAs could be potential therapeutic targets.

A differential microRNA expression has been reported in patients with diabetic retinopathy as compared to controls. Animal and in vitro studies on RECs too showed altered retinal microRNA profile in diabetic animals and RECs treated with high glucose (35). Zampetaki et al. (101) showed that miR-27b and miR-320a increased risk of diabetic retinopathy by repressing antiangiogenic thrombospondin-1. Qin et al. have reported decreased miR-20b and correlated increase in its target genes VEGF and AKT3 in the retina and RECs in diabetic rats. These authors suggested that hyperglycemia-induced changes in retinal tissues were mediated by miR-20b via modulating VEGF and AKT3 in diabetic retinopathy (102). Similarly miR-15a too has been found to be protective toward developing diabetic retinopathy by inhibiting pro-inflammatory and pro-angiogenic pathways through its target genes ASM and VEGF-A (103). In a recent study, Zhou et al. (104) showed that transgenic mice over expressing let-7 show features similar to non-proliferative diabetic retinopathy suggesting its pathological role in nonproliferative diabetic retinopathy.

In addition, genetic variants in microRNA genes, such as miR-4513 rs2168518, miR-499 rs3746444, miR-196a2 rs11614913, and miR-423 rs6505162, have also been shown to be associated with the risk of cardiovascular complication of diabetes (105).

Taken together, available literature shows a definitive role of microRNAs, specifically targeting pro-angiogenic and proinflammatory genes in pathogenesis of diabetic retinopathy, thus providing their therapeutic potential in preventing and treatment of diabetic retinopathy.

\section{MICRORNAS AS BIOMARKERS OF DIABETIC VASCULAR COMPLICATIONS}

As serum levels of different miRNAs have been shown to be elevated in cardiovascular complication of diabetes, they could serve as sensitive and cost-effective biomarkers for these conditions. For example, the expression levels of seven diabetes-related miRNAs (miR-9, miR-29a, miR-30d, miR-34a, miR-124a, miR-146a, and miR-375) in serum were shown to be significantly elevated in T2DM subjects compared with pre-diabetes and/or normal glucose tolerance suggesting that during the pathogenesis of T2DM, the peripheral diabetesrelated miRNAs have not changed significantly from susceptible individual with normal glucose tolerance at pre-diabetic stage (106). miR-1 and miR-133a have been found to be good predictors of myocardial steatosis in diabetic patients (105). The fact that miR-1 and miR-133a are poorly associated with other clinical, biochemical, metabolic, hemodynamic, and cardiac parameters, and even with verified absence of clinically evident myocardial ischemia and/or damage supports the hypothesis that these miRNAs are independent predictors of myocardial steatosis.

miR-21, miR-29a/b/c, and miR-192 could reflect DN pathogenesis and serve as biomarkers during $\mathrm{DN}$ progression as there levels were significantly enriched in the overt proteinuria group compared with microalbuminuria and/or overt proteinuria groups. Authors observed that miR-192 suppressed the translation of SIP1/E-box repressors ZEB2, leading to elevated collagen deposition in vivo indicating a role of miR-192 in the development of the matrix accumulation observed in DN. Whereas, miR-21 prevented mesangial hypertrophy by targeting the PTEN/PI3K/ AKT pathway and miR-29 was negatively regulated by TGF- $\beta 1$ via SMAD3 signaling pathway, thereby promoting collagen matrix expression (107).

Some microRNAs have been found significantly increased in blood samples of diabetic patients with retinopathy; for example, Qing et al. (108) have reported that circulating miR-21, 
miR-181c, and miR-1179 together could be good biomarkers for differentiating between proliferative and non-proliferative retinopathy. Barutta et al. (109) recently reported that circulating miR-126 levels were significantly lower in diabetic patients as compared to controls and were associated with both micro- and macrovascular complications, especially with proliferative retinopathy In a large cohort of type 1 diabetic subjects. Circulating microRNAs as biomarkers of diabetes-induced cardiomyopathy have also been reviewed recently (110).

Exosomes are small extracellular vesicles present in blood and urine is rich in microRNAs and is being investigated as potential disease markers. Mohan et al. (111) showed that urinary exosomal microRNAs 451-5p levels increased and correlated with renal damage in diabetic rats and suggested these to be useful as early biomarkers of diabetic nephropathy. Thus, microRNAs show promising potential as biomarkers for vascular complications of diabetes.

\section{MICRORNAS AS THERAPEUTICS IN MICROVASCULAR COMPLICATIONS OF DIABETES}

MicroRNAs have been explored for their potential as new therapeutic targets in diabetes vascular complications; for example, Kovacs et al. (82) showed that miR-146 through its inhibition of NF-k $\beta$ activation could be a potential therapeutic target in cardiovascular complication of diabetes. miR-130a is shown to improve EPCs function by negatively regulating RUNX3 and through ERK/VEGF and AKT pathways and could have a potential use in improving endothelial function (112). Downregulation of miR-200b has been implicated in Glucoseinduced augmented vascular endothelial growth factor (VEGF) production through histone $\mathrm{H} 3$ lysine-27 trimethylation (113). Thus, methyltransferase inhibitors like COMT inhibitor could be used to control VEGF augmentation by upregulation of miR-200b. On similar grounds, in a recent study, vitamin $B_{3}$ and nicotinic acid have been shown to have a protective effect in diabetic retinopathy by upregulating miR-126 (114). miR34 family modulates changes in proliferation and migration of retinal pigment epithelial cells through downregulation of leucine-rich repeat-containing G-protein coupled receptor 4 (LGR4) expressions, indicating G protein (heterotrimeric) inhibitors as potential therapeutics (115). Apart from this, miR21 is an important miRNA frequently upregulated in T2DM and cardiovascular complication of diabetes (116). miR-21 targets SMAD7 pathway and also blocks the expression of PDCD4 and thereby, suppress activation of the TGF- $\beta$ and NF- $\kappa B$ signaling pathways. Since miR-21 is upregulated in cells related to diabetic complications, their exclusive molecular signatures can be used as prognosis, diagnosis, and therapeutic targets. Sekar et al. (79) have also shown that targeting miR-21 by synthetic anti-miRNA oligonucleotides (AMOs) with 2-O-methylmodification effectively inhibited the miRNA 21 in cell culture and xenograft mouse models. In addition to this, antisense-RNA, miRNAs mimics, and tumor suppressor miRNAs could be also used to inhibit the expression of miR-21.

\section{LONG NON-CODING RNAs AND VASCULAR COMPLICATIONS OF DIABETES}

Long non-coding RNAs (lncRNAs) are >200-nt-long noncoding RNAs and are increasingly being recognized as important gene regulators. lncRNAs repress gene expression by binding to specific DNA/RNA or protein moieties (80). For example, they can bind to miRNAs and thereby prevent their binding to target mRNAs and, hence, gene expression (81) or they may regulate activity of regulatory proteins by altering their affinity or cellular localization for other proteins (83). Aberrant expression of lncRNAs has been implicated in pathophysiology of several diseases such as tumorigenesis and cardiovascular diseases; however, their role in vascular complications of diabetes remains largely unknown. Recent studies have identified several lncRNAs with potential role in diabetic nephropathy, retinopathy, neuropathy, and cardiomyopathy.

Data on lncRNAs in diabetic cardiomyopathy are sparse. Zhang et al. (117) recently reported increased expression of lncRNAs MALAT1 in the heart tissue of diabetic rats, and observed that its inhibition Improved left ventricular function, by attenuating cardiomyocyte apoptosis. A downregulation of lncRNA H19 has been also seen in diabetic hearts and it has been shown to increase expression of miRNA-675 and downregulation of its target VDAC1 leading to decreased cardiomyocyte apoptosis of cardiomyocytes in high glucose milieu (118). Zhuo et al. (119) have recently showed that $\mathrm{H} 19$ also inhibited autophagy in glucosetreated cardiomyocytes by silencing pro-autophagy DIRAS3. Inc $\mathrm{H} 19$ has been suggested as a potential biomarker and therapeutic target for diabetic cardiomyopathy. However, more research is needed to explore the potential role of lncRNAs in diabetic cardiomyopathy.

Wang et al. (120) reported downregulation of CYP4B1-PS1-001 in both in early stages of diabetic nephropathy and suggested its role in mesangial cell proliferation and fibrosis. Alvarez et al. (100) earlier showed that a long non-coding RNA, the plasmacytoma variant translocation 1 (PVT1), increased fibronectin 1 (FN1) ECM accumulation in the glomeruli under hyperglycemic conditions, suggesting its role in diabetic nephropathy. They recently reported that miR-1207-5p, a PVT1-derived microRNA, was also independently involved in pathogenesis of diabetic nephropathy. Similarly lncRNA ENSMUST00000147869 associated with Cyp4a12a has been shown to mediate diabetic nephropathy by increasing proliferation and fibrosis of mesangial cells (121). Several other lncRNAs, such as MALAT1 (122), myocardial infarction-associated transcript (MIAT) (123) and lnc-MGC, have been found to be dysregulated in diabetes-induced renal injury and are potential therapeutic targets for treating diabetesinduced nephropathy.

IncRNA-RNCR3 has been implicated in diabetes-induced retinopathy. Liu et al. (67) recently showed that lncRNA-RNCR3 knockdown decreased cytokine levels, retinal cell apoptosis, improved visual function, and inhibited retinal reactive gliosis in diabetic animals, indicating its role in diabetes-induced neurodegeneration. Shan et al. have increase in RNCR3 levels following high glucose stress both in vitro and in vivo. They observed 
that RNCR3 knockdown inhibited RECs proliferation, and cell migration and tube formation in vitro and improved endothelial function in vivo (124) via RNCR3/KLF2/miR-185-5p pathway, suggesting RNCR3 inhibition as a therapeutic option in treating diabetic retinal abnormalities.

The studies done so far indicate that lncRNAs are important mediators of various vascular complications of diabetes and potential therapeutic targets and need to be explored further.

\section{CONCLUDING REMARKS}

Metabolic disorders such as diabetes are due to cumulative interactive effects of genetic and environmental factors. These effects are primarily induced by diabetes-associated factors, such as hyperglycemia, oxidative stress, inflammation, obesity, and so on, and are manifested as epigenetic changes in the genome. These epigenetic changes include DNA methylation, histone methylation and acetylation, deregulated expression of microRNAs and lncRNAs etc. and are responsible for altered gene expression of the key regulatory pathways mediating diabetes-associated vascular complications and also are major contributors to metabolic memory associated with diabetes. Thus, study of epigenetic mechanisms assumes a significant role in elucidating pathophysiology of diabetes and its complications. However, our understanding of these mechanisms is incomplete and awaits translational application. Further research focus is needed to elucidate the mechanisms especially with respect to non-coding RNAs and chromatin structure. The information being generated in microRNAs and lncRNAs shows that we are at threshold of unveiling of important biological role of these molecules in disease etiology, pathology, progression,

\section{REFERENCES}

1. Nature. Access: Moving AHEAD with an International Human Epigenome Project (2017). Available from: http://www.nature.com/nature/journal/v454/ n7205/full/454711a.html

2. Centers for Disease Control and Prevention. National Diabetes Fact Sheet: National Estimates and General Information on Diabetes and Prediabetes in the United States, 2011. Atlanta GA: United States Department of Health and Human Services, Centers for Disease Control and Prevention (2011). 201 p. Available from: http://www.diabetesincontrol.com/wp-content/uploads/ PDF/ndep_diabetes_facts_2011.pdf

3. Jayaraman S. Epigenetics of autoimmune diabetes. Epigenomics (2011) 3:639-48. doi:10.2217/epi.11.78

4. Ling C, Groop L. Epigenetics: a molecular link between environmental factors and type 2 diabetes. Diabetes (2009) 58:2718-25. doi:10.2337/ db09-1003

5. Togliatto G, Dentelli P, Brizzi MF. Skewed epigenetics: an alternative therapeutic option for diabetes complications. J Diabetes Res (2015) 2015:373708. doi:10.1155/2015/373708

6. Portela A, Esteller M. Epigenetic modifications and human disease. Nat Biotechnol (2010) 28:1057-68. doi:10.1038/nbt.1685

7. Wang Z, Gucek M, Hart GW. Cross-talk between GlcNAcylation and phosphorylation: site-specific phosphorylation dynamics in response to globally elevated O-GlcNAc. Proc Natl Acad Sci U S A (2008) 105:13793-8. doi:10.1073/pnas.0806216105

8. Marsh SA, Collins HE, Chatham JC. Protein O-GlcNAcylation and cardiovascular (patho) physiology. J Biol Chem (2014) 289:34449-56. doi:10.1074/ jbc.R114.585984 and therapeutics, besides being non-invasive diagnostic and prognostic biomarkers of vascular complications, such as nephropathy and cardiomyopathy.

To gain a deeper understanding of T2DM and its associated microvascular complications, an incorporation of a range of novel tools and techniques, such as RNAseq, transcriptomics, metabolomics, epigenomic profiling, and chromatin $3 \mathrm{D}$ mapping, is needed to be integrated in diabetes research. Tissue- and cellspecific profiling of methylation levels and histone modifications of major pathophysiological genes would increase our understanding of the pathology of T2DM and associated complications. Elucidation of association between epigenetic modulations of the genome involved in microvascular complication with those of macrovascular complications of diabetes is also needed. The knowledge gained through epigenetics gene expression alteration in diabetic cardiomyopathy will provide better approaches in attenuating hyperglycemia-induced damage to the heart and other affected organs, such as kidney and brain. Thus, elucidation of epigenetic mechanisms in conjunction with environmental and genetic factors would fine tune the understanding of pathophysiology of diabetic cardiomyopathy. And, epigenetic factors could provide a wholesome picture of the role of genes and their expression in T2DM and its micro as well as macrovascular complications.

\section{AUTHOR CONTRIBUTIONS}

MK: checking, editing, re-writing, data approval, and guarantor of work. BC: data collection, writing, and reference updation. SR: checking, editing, data collection, writing, and reference updation.

9. Cox EJ, Marsh SA. Exercise and diabetes have opposite effects on the assembly and O-GlcNAc modification of the mSin3A/HDAC1/2 complex in the heart. Cardiovasc Diabetol (2013) 12:101. doi:10.1186/14752840-12-101

10. Xu Z, Tong Q, Zhang Z, Wang S, Zheng Y, Liu Q, et al. Inhibition of HDAC3 prevents diabetic cardiomyopathy in OVE26 mice via epigenetic regulation of DUSP5-ERK1/2 pathway. Clin Sci (2017) 131(15):1841-57. doi:10.1042/ CS20170064

11. Li X, Li C, Sun G. Histone acetylation and its modifiers in the pathogenesis of diabetic nephropathy. JDiabetes Res (2016) 2016:4065382. doi:10.1155/2016/4065382

12. Yuan H, Reddy MA, Sun G, Lanting L, Wang M, Kato M, et al. Involvement of p300/CBP and epigenetic histone acetylation in TGF- $\beta 1$-mediated gene transcription in mesangial cells. Am J Physiol Renal Physiol (2013) 304:F601-13. doi:10.1152/ajprenal.00523.2012

13. Noh H, Oh EY, Seo JY, Yu MR, Kim YO, Ha H, et al. Histone deacetylase-2 is a key regulator of diabetes-and transforming growth factor- $\beta 1$-induced renal injury. Am J Physiol Renal Physiol (2009) 297:F729-39. doi:10.1152/ ajprenal.00086.2009

14. Wang X, Liu J, Zhen J, Zhang C, Wan Q, Liu G, et al. Histone deacetylase 4 selectively contributes to podocyte injury in diabetic nephropathy. Kidney Int (2014) 86:712-25. doi:10.1038/ki.2014.111

15. Wang C, You Q, Cao X, Guo H, Gao X, Peng X. Micro RNA-19a suppresses interleukin-10 in peripheral B cells of patients with diabetic retinopathy. Am J Transl Res (2017) 9:1410-7.

16. Rodenhiser D, Mann M. Epigenetics and human disease: translating basic biology into clinical applications. Can Med Assoc J (2006) 174:341-8. doi:10.1503/cmaj.050774 
17. Miao F, Gonzalo IG, Lanting L, Natarajan R. In vivo chromatin remodeling events leading to inflammatory gene transcription under diabetic conditions. J Biol Chem (2004) 279:18091-7. doi:10.1074/jbc.M311786200

18. Sterns JD, Smith CB, Steele JR, Stevenson KL, Gallicano GI. Epigenetics and type II diabetes mellitus: underlying mechanisms of prenatal predisposition. Front Cell Dev Biol (2014) 2:15. doi:10.3389/fcell.2014.00015

19. Yun J-M, Jialal I, Devaraj S. Epigenetic regulation of high glucose-induced proinflammatory cytokine production in monocytes by curcumin. J Nutr Biochem (2011) 22:450-8. doi:10.1016/j.jnutbio.2010.03.014

20. Herranz D, Muñoz-Martin M, Cañamero M, Mulero F, Martinez-Pastor B, Fernandez-Capetillo O, et al. Sirtl improves healthy ageing and protects from metabolic syndrome-associated cancer. Nat Commun (2010) 1:3. doi:10.1038/ncomms1001

21. Guarente L. Sirtuins, aging, and medicine. N Engl J Med (2011) 364:2235-44. doi:10.1056/NEJMra1100831

22. Inoue H, Ogawa W, Ozaki M, Haga S, Matsumoto M, Furukawa K, et al. Role of STAT-3 in regulation of hepatic gluconeogenic genes and carbohydrate metabolism in vivo. Nat Med (2004) 10:168-74. doi:10.1038/nm980

23. Orimo M, Minamino T, Miyauchi H, Tateno K, Okada S, Moriya J, et al. Protective role of SIRT1 in diabetic vascular dysfunction. Arterioscler Thromb Vasc Biol (2009) 29:889-94. doi:10.1161/ATVBAHA.109.185694

24. Bagul PK, Dinda AK, Banerjee SK. Effect of resveratrol on sirtuins expression and cardiac complications in diabetes. Biochem Biophys Res Commun (2015) 468:221-7. doi:10.1016/j.bbrc.2015.10.126

25. Liang F, Kume S, Koya D. SIRT1 and insulin resistance. Nat Rev Endocrinol (2009) 5:367-73. doi:10.1038/nrendo.2009.101

26. D’Onofrio N, Vitiello M, Casale R, Servillo L, Giovane A, Balestrieri ML. Sirtuins in vascular diseases: emerging roles and therapeutic potential. Biochim Biophys Acta (2015) 1852:1311-22. doi:10.1016/j.bbadis.2015.03.001

27. Mortuza R, Chen S, Feng B, Sen S, Chakrabarti S. High glucose induced alteration of SIRTs in endothelial cells causes rapid aging in a p300 and FOXO regulated pathway. PLoS One (2013) 8(1):e54514. doi:10.1371/journal. pone. 0054514

28. Li P, Zhang L, Zhou C, Lin N, Liu A. Sirt 1 activator inhibits the AGE-induced apoptosis and p53 acetylation in human vascular endothelial cells. J Toxicol Sci (2015) 40:615-24. doi:10.2131/jts.40.615

29. Mortuza R, Feng B, Chakrabarti S. SIRT1 reduction causes renal and retinal injury in diabetes through endothelin 1 and transforming growth factor $\beta 1$. J Cell Mol Med (2015) 19:1857-67. doi:10.1111/jcmm.12557

30. Mishra M, Flaga J, Kowluru RA. Molecular mechanism of transcriptional regulation of matrix metalloproteinase- 9 in diabetic retinopathy. J Cell Physiol (2016) 231:1709-18. doi:10.1002/jcp.25268

31. Chang M, Zhang B, Tian Y, Hu M, Zhang G, Di Z, et al. AGEs decreased SIRT3 expression and SIRT3 activation protected AGEs-induced EPCs' dysfunction and strengthened anti-oxidant capacity. Inflammation (2017) 40:473-85. doi:10.1007/s10753-016-0493-1

32. Thandavarayan RA, Garikipati VNS, Joladarashi D, Suresh Babu S, Jeyabal P, Verma SK, et al. Sirtuin-6 deficiency exacerbates diabetes-induced impairment of wound healing. Exp Dermatol (2015) 24:773-8. doi:10.1111/ exd.12762

33. Miao F, Wu X, Zhang L, Yuan Y-C, Riggs AD, Natarajan R. Genome-wide analysis of histone lysine methylation variations caused by diabetic conditions in human monocytes. J Biol Chem (2007) 282:13854-63. doi:10.1074/ jbc.M609446200

34. Okabe J, Orlowski C, Balcerczyk A, Tikellis C, Thomas MC, Cooper ME, et al. Distinguishing hyperglycemic changes by Set7 in vascular endothelial cells novelty and significance. Circ Res (2012) 110:1067-76. doi:10.1161/ CIRCRESAHA.112.266171

35. Pirola L, Balcerczyk A, Tothill RW, Haviv I, Kaspi A, Lunke S, et al. Genomewide analysis distinguishes hyperglycemia regulated epigenetic signatures of primary vascular cells. Genome Res (2011) 21:1601-15. doi:10.1101/ gr.116095.110

36. Zhong Q, Kowluru RA. Epigenetic changes in mitochondrial superoxide dismutase in the retina and the development of diabetic retinopathy. Diabetes (2011) 60:1304-13. doi:10.2337/db10-0133

37. Tateishi K, Okada Y, Kallin EM, Zhang Y. Role of Jhdm2a in regulating metabolic gene expression and obesity resistance. Nature (2009) 458:757-61. doi:10.1038/nature07777
38. Özkan H, Aydın A, Demir N, Erci T, Büyükgebiz A. Associations of IGF-I, IGFBP-1 and IGFBP-3 on intrauterine growth and early catch-up growth. Neonatology (1999) 76:274-82. doi:10.1159/000014169

39. Yu X-Y, Geng Y-J, Liang J-L, Zhang S, Lei H-P, Zhong S-L, et al. High levels of glucose induce "metabolic memory" in cardiomyocyte via epigenetic histone H3 lysine 9 methylation. Mol Biol Rep (2012) 39:8891-8. doi:10.1007/ s11033-012-1756-z

40. Kato M, Natarajan R. Diabetic nephropathy - emerging epigenetic mechanisms. Nat Rev Nephrol (2014) 10:517-30. doi:10.1038/nrneph.2014.116

41. Li X, Li C, Li X, Cui P, Li Q, Guo Q, et al. Involvement of histone lysine methylation in $\mathrm{p} 21$ gene expression in rat kidney in vivo and rat mesangial cells in vitro under diabetic conditions. J Diabetes Res (2016) 2016:3853242. doi:10.1155/2016/3853242

42. Zhong Q, Kowluru RA. Epigenetic modification of Sod2 in the development of diabetic retinopathy and in the metabolic memory: role of histone methylationhistone methylation and diabetic retinopathy. Invest Ophthalmol Vis Sci (2013) 54:244-50. doi:10.1167/iovs.12-10854

43. Kim D-I, Park M-J, Choi J-H, Kim I-S, Han H-J, Yoon K-C, et al. PRMT1 and PRMT4 regulate oxidative stress-induced retinal pigment epithelial cell damage in SIRT1-dependent and SIRT1-independent manners. Oxid Med Cell Longev (2015) 2015:617919. doi:10.1155/2015/617919

44. Wang W, Sidoli S, Zhang W, Wang Q, Wang L, Jensen ON, et al. Abnormal levels of histone methylation in the retinas of diabetic rats are reversed by minocycline treatment. Sci Rep (2017) 7:45103. doi:10.1038/srep45103

45. Kowluru RA, Santos JM, Mishra M. Epigenetic modifications and diabetic retinopathy. Biomed Res Int (2013) 2013:635284. doi:10.1155/2013/635284

46. Wang D, Fang C, Zong NC, Liem DA, Cadeiras M, Scruggs SB, et al. Regulation of acetylation restores proteolytic function of diseased myocardium in mouse and human. Mol Cell Proteomics (2013) 12:3793-802. doi:10.1074/mcp.M113.028332

47. Kao Y-H, Liou J-P, Chung C-C, Lien G-S, Kuo C-C, Chen S-A, et al. Histone deacetylase inhibition improved cardiac functions with direct antifibrotic activity in heart failure. Int J Cardiol (2013) 168:4178-83. doi:10.1016/j. ijcard.2013.07.111

48. Eom GH, Nam YS, Oh JG, Choe N, Min H-K, Yoo E-K, et al. Regulation of acetylation of histone deacetylase 2 by p300/CBP-associated factor/histone deacetylase 5 in the development of cardiac hypertrophy. Circ Res (2014) 114(7):1133-43. doi:10.1161/CIRCRESAHA.114.303429

49. Christensen DP, Dahllöf M, Lundh M, Rasmussen DN, Nielsen MD, Billestrup N, et al. Histone deacetylase (HDAC) inhibition as a novel treatment for diabetes mellitus. Mol Med (2011) 17:378. doi:10.2119/ molmed.2011.00021

50. Chen Y, Du J, Zhao YT, Zhang L, Lv G, Zhuang S, et al. Histone deacetylase (HDAC) inhibition improves myocardial function and prevents cardiac remodeling in diabetic mice. Cardiovasc Diabetol (2015) 14:99. doi:10.1186/ s12933-015-0262-8

51. Lee T-I, Kao Y-H, Tsai W-C, Chung C-C, Chen Y-C, Chen Y-J. HDAC inhibition modulates cardiac PPARs and fatty acid metabolism in diabetic cardiomyopathy. PPAR Res (2016) 2016:5938740. doi:10.1155/2016/ 5938740

52. Gilbert RE, Huang Q, Thai K, Advani SL, Lee K, Yuen DA, et al. Histone deacetylase inhibition attenuates diabetes-associated kidney growth: potential role for epigenetic modification of the epidermal growth factor receptor. Kidney Int (2011) 79:1312-21. doi:10.1038/ki.2011.39

53. Advani A, Huang Q, Thai K, Advani SL, White KE, Kelly DJ, et al. Longterm administration of the histone deacetylase inhibitor vorinostat attenuates renal injury in experimental diabetes through an endothelial nitric oxide synthase-dependent mechanism. Am J Pathol (2011) 178:2205-14. doi:10.1016/j.ajpath.2011.01.044

54. Khan S, Jena G, Tikoo K. Sodium valproate ameliorates diabetes-induced fibrosis and renal damage by the inhibition of histone deacetylases in diabetic rat. Exp Mol Pathol (2015) 98:230-9. doi:10.1016/j.yexmp.2015.01.003

55. Dong W, Jia Y, Liu X, Zhang H, Li T, Huang W, et al. Sodium butyrate activates NRF2 to ameliorate diabetic nephropathy possibly via inhibition of HDAC. J Endocrinol (2017) 232:71-83. doi:10.1530/JOE-16-0322

56. Hakami NY, Dusting GJ, Peshavariya HM. Trichostatin A, a histone deacetylase inhibitor suppresses NADPH oxidase 4-derived redox signalling and angiogenesis. J Cell Mol Med (2016) 20:1932-44. doi:10.1111/jcmm.12885 
57. Cao DJ, Wang ZV, Battiprolu PK, Jiang N, Morales CR, Kong Y, et al. Histone deacetylase (HDAC) inhibitors attenuate cardiac hypertrophy by suppressing autophagy. Proc Natl Acad Sci U S A (2011) 108:4123-8. doi:10.1073/ pnas. 1015081108

58. Park JH, Stoffers DA, Nicholls RD, Simmons RA. Development of type 2 diabetes following intrauterine growth retardation in rats is associated with progressive epigenetic silencing of Pdxl. J Clin Invest (2008) 118:2316-24. doi:10.1172/JCI33655

59. Johnson E, Marsh S. Anti-diabetic effects of class 1 histone deacetylase inhibition in a rodent model of type 2 diabetes mellitus. FASEB J (2016) 30:1273.6.

60. Hirschey MD, Shimazu T, Jing E, Grueter CA, Collins AM, Aouizerat B, et al. SIRT3 deficiency and mitochondrial protein hyperacetylation accelerate the development of the metabolic syndrome. Mol Cell (2011) 44:177-90. doi:10.1016/j.molcel.2011.07.019

61. Nasrin N, Wu X, Fortier E, Feng Y, Bare OC, Chen S, et al. SIRT4 regulates fatty acid oxidation and mitochondrial gene expression in liver and muscle cells. J Biol Chem (2010) 285:31995-2002. doi:10.1074/jbc.M110.124164

62. Zheng J, Cheng J, Zhang Q, Xiao X. Novel insights into DNA methylation and its critical implications in diabetic vascular complications. Biosci Rep (2017) 37:BSR20160611. doi:10.1042/BSR20160611

63. El-Osta A, Brasacchio D, Yao D, Pocai A, Jones PL, Roeder RG, et al. Transient high glucose causes persistent epigenetic changes and altered gene expression during subsequent normoglycemia. J Exp Med (2008) 205:2409-17. doi: $10.1084 /$ jem. 20081188

64. Movassagh M, Choy M-K, Goddard M, Bennett MR, Down TA, Foo RS-Y. Differential DNA methylation correlates with differential expression of angiogenic factors in human heart failure. PLoS One (2010) 5:e8564. doi:10.1371/journal.pone.0008564

65. Mönkemann H, De Vriese AS, Blom HJ, Kluijtmans LAJ, Heil SG, Schild HH, et al. Early molecular events in the development of the diabetic cardiomyopathy. Amino Acids (2002) 23:331-6. doi:10.1007/s00726-001-0146-y

66. Zhong J, Agha G, Baccarelli AA. The role of DNA methylation in cardiovascular risk and disease. Circ Res (2016) 118:119-31. doi:10.1161/ CIRCRESAHA.115.305206

67. Liu Z-Z, Zhao X-Z, Zhang X-S, Zhang M. Promoter DNA demethylation of Keapl gene in diabetic cardiomyopathy. Int J Clin Exp Pathol (2014) 7:8756-62.

68. Vecellio M, Spallotta F, Nanni S, Colussi C, Cencioni C, Derlet A, et al. The histone acetylase activator pentadecylidenemalonate $1 \mathrm{~b}$ rescues proliferation and differentiation in the human cardiac mesenchymal cells of type 2 diabetic patients. Diabetes (2014) 63:2132-47. doi:10.2337/ db13-0731

69. Cheng Y, Liu G, Pan Q, Guo S, Yang X. Elevated expression of liver X receptor alpha $(\mathrm{LXR} \alpha)$ in myocardium of streptozotocin-induced diabetic rats. Inflammation (2011) 34:698-706. doi:10.1007/s10753-010-9281-5

70. Kao Y-H, Chen Y-C, Cheng C-C, Lee T-I, Chen Y-J, Chen S-A. Tumor necrosis factor- $\alpha$ decreases sarcoplasmic reticulum Ca2+-ATPase expressions via the promoter methylation in cardiomyocytes. Crit Care Med (2010) 38:217-22. doi:10.1097/CCM.0b013e3181b4a854

71. Bell CG, Teschendorff AE, Rakyan VK, Maxwell AP, Beck S, Savage DA. Genome-wide DNA methylation analysis for diabetic nephropathy in type 1 diabetes mellitus. BMC Med Genomics (2010) 3:33. doi:10.1186/ 1755-8794-3-33

72. Peng R, Liu H, Peng H, Zhou J, Zha H, Chen X, et al. Promoter hypermethylation of let-7a-3 is relevant to its down-expression in diabetic nephropathy by targeting UHRF1. Gene (2015) 570:57-63. doi:10.1016/j.gene.2015.05.073

73. Sapienza C, Lee J, Powell J, Erinle O, Yafai F, Reichert J, et al. DNA methylation profiling identifies epigenetic differences between diabetes patients with ESRD and diabetes patients without nephropathy. Epigenetics (2011) 6:20-8. doi:10.4161/epi.6.1.13362

74. Agardh E, Lundstig A, Perfilyev A, Volkov P, Freiburghaus T, Lindholm E, et al. Genome-wide analysis of DNA methylation in subjects with type 1 diabetes identifies epigenetic modifications associated with proliferative diabetic retinopathy. BMC Med (2015) 13:182. doi:10.1186/s12916015-0421-5

75. Mishra M, Kowluru RA. Epigenetic modification of mitochondrial DNA in the development of diabetic retinopathy methylation of mtDNA in diabetic retinopathy. Invest Ophthalmol Vis Sci (2015) 56:5133-42. doi:10.1167/ iovs.15-16937

76. Kowluru RA, Shan Y, Mishra M. Dynamic DNA methylation of matrix metalloproteinase-9 in the development of diabetic retinopathy. Lab Invest (2016) 96(10):1040-9. doi:10.1038/labinvest.2016.78

77. Ito K, Hanazawa T, Tomita K, Barnes P, Adcock IM. Oxidative stress reduces histone deacetylase 2 activity and enhances IL- 8 gene expression: role of tyrosine nitration. Biochem Biophys Res Commun (2004) 315:240-5. doi:10.1016/j.bbrc.2004.01.046

78. Jamaluddin MS, Yang X, Wang H. Hyperhomocysteinemia, DNA methylation and vascular disease. Clin Chem Lab Med (2007) 45:1660-6. doi:10.1515/ CCLM.2007.350

79. Sekar D, Saravanan S, Karikalan K, Thirugnanasambantham K, Lalitha P, IH Islam V. Role of microRNA 21 in mesenchymal stem cell (MSC) differentiation: a powerful biomarker in MSCs derived cells. Curr Pharm Biotechnol (2015) 16:43-8. doi:10.2174/138920101601150105100851

80. Moura J, Børsheim E, Carvalho E. The role of microRNAs in diabetic complications-special emphasis on wound healing. Genes (2014) 5:926-56. doi:10.3390/genes5040926

81. de Gonzalo-Calvo D, van der Meer RW, Rijzewijk LJ, Smit JWA, RevueltaLopez E, Nasarre L, et al. Serum microRNA-1 and microRNA-133a levels reflect myocardial steatosis in uncomplicated type 2 diabetes. Sci Rep (2017) 7:47. doi:10.1038/s41598-017-00070-6

82. Kovacs B, Lumayag S, Cowan C, Xu S. MicroRNAs in early diabetic retinopathy in streptozotocin-induced diabetic rats. Invest Ophthalmol Vis Sci (2011) 52:4402-9. doi:10.1167/iovs.10-6879

83. Chien H-Y, Chen C-Y, Chiu Y-H, Lin Y-C, Li W-C. Differential microRNA profiles predict diabetic nephropathy progression in Taiwan. Int J Med Sci (2016) 13:457. doi:10.7150/ijms.15548

84. Liu X, Liu S. Role of microRNAs in the pathogenesis of diabetic cardiomyopathy. Biomed Rep (2017) 6:140-5. doi:10.3892/br.2017.841

85. Raut SK, Kumar A, Singh GB, Nahar U, Sharma V, Mittal A, et al. miR-30c mediates upregulation of $\mathrm{Cdc} 42$ and Pak1 in diabetic cardiomyopathy. Cardiovasc Ther (2015) 33:89-97. doi:10.1111/1755-5922.12113

86. Raut SK, Singh GB, Rastogi B, Saikia UN, Mittal A, Dogra N, et al. miR-30c and miR-181a synergistically modulate $\mathrm{p} 53-\mathrm{p} 21$ pathway in diabetes induced cardiac hypertrophy. Mol Cell Biochem (2016) 417:191-203. doi:10.1007/ s11010-016-2729-7

87. Singh GB, Raut SK, Khanna S, Kumar A, Sharma S, Prasad R, et al. MicroRNA200c modulates DUSP-1 expression in diabetes-induced cardiac hypertrophy. Mol Cell Biochem (2017) 424:1-11. doi:10.1007/s11010-016-2838-3

88. Ning F, Qiao Q, Tuomilehto J, Hammar N, Ho SY, Söderberg S, et al. Does abnormal insulin action or insulin secretion explain the increase in prevalence of impaired glucose metabolism with age in populations of different ethnicities? Diabetes Metab Res Rev (2010) 26:245-53. doi:10.1002/ dmrr. 1078

89. Duan Y, Zhou B, Su H, Liu Y, Du C. miR-150 regulates high glucose-induced cardiomyocyte hypertrophy by targeting the transcriptional co-activator p300. Exp Cell Res (2013) 319:173-84. doi:10.1016/j.yexcr.2012.11.015

90. Shen E, Diao X, Wang X, Chen R, Hu B. MicroRNAs involved in the mitogen-activated protein kinase cascades pathway during glucose-induced cardiomyocyte hypertrophy. Am J Pathol (2011) 179:639-50. doi:10.1016/j. ajpath.2011.04.034

91. Kuwabara Y, Horie T, Baba O, Watanabe S, Nishiga M, Usami S, et al. MicroRNA-451 exacerbates lipotoxicity in cardiac myocytes and high-fat diet-induced cardiac hypertrophy in mice through suppression of the LKB1/ AMPK pathway. Circ Res (2015) 116:279-88. doi:10.1161/CIRCRESAHA. 116.304707

92. Liu S, Li W, Xu M, Huang H, Wang J, Chen X. Micro-RNA 21 targets dual specific phosphatase 8 to promote collagen synthesis in high glucose-treated primary cardiac fibroblasts. Can J Cardiol (2014) 30:1689-99. doi:10.1016/j. cjca.2014.07.747

93. Thum T, Gross C, Fiedler J, Fischer T, Kissler S, Bussen M, et al. MicroRNA-21 contributes to myocardial disease by stimulating MAP kinase signalling in fibroblasts. Nature (2008) 456:980-4. doi:10.1038/nature07511

94. Kumar A, Raut SK, Saikia UN, Sharma R, Khullar M. Microrna-21 contributes to diabetic cardiomyopathy associated cardiac fibrosis. Circulation (2011) 124:A15227. 
95. Chen J, Wang R, Zhang K, Chen L-B. Long non-coding RNAs in non-small cell lung cancer as biomarkers and therapeutic targets. J Cell Mol Med (2014) 18:2425-36. doi:10.1111/jcmm.12431

96. Zhao Y, Samal E, Srivastava D. Serum response factor regulates a musclespecific microRNA that targets Hand2 during cardiogenesis. Nature (2005) 436:214-20. doi:10.1038/nature03817

97. Shan Z-X, Lin Q-X, Deng C-Y, Zhu J-N, Mai L-P, Liu J-L, et al. miR-1/ miR-206 regulate Hsp60 expression contributing to glucose-mediated apoptosis in cardiomyocytes. FEBS Lett (2010) 584:3592-600. doi:10.1016/j. febslet.2010.07.027

98. Li X, Wang H, Yao B, Xu W, Chen J, Zhou X. IncRNA H19/miR-675 axis regulates cardiomyocyte apoptosis by targeting VDAC1 in diabetic cardiomyopathy. Sci Rep (2016) 6:36340. doi:10.1038/srep36340

99. Kato M, Natarajan R. MicroRNAs in diabetic nephropathy: functions, biomarkers, and therapeutic targets. Ann N Y Acad Sci (2015) 1353:72-88. doi: $10.1111 /$ nyas. 12758

100. Alvarez ML, Khosroheidari M, Eddy E, Kiefer J. Role of microRNA 1207-5P and its host gene, the long non-coding RNA Pvt1, as mediators of extracellular matrix accumulation in the kidney: implications for diabetic nephropathy. PLoS One (2013) 8:e77468. doi:10.1371/journal.pone.0077468

101. Zampetaki A, Willeit P, Burr S, Yin X, Langley SR, Kiechl S, et al. Angiogenic microRNAs linked to incidence and progression of diabetic retinopathy in type 1 diabetes. Diabetes (2016) 65(1):216-27. doi:10.2337/db15-0389

102. Qin B, Liu J, Liu S, Li B, Ren J. miR-20b targets AKT3 and modulates vascular endothelial growth factor-mediated changes in diabetic retinopathy. Acta Biochim Biophys Sin (2016) 48:732-40. doi:10.1093/abbs/gmw065

103. Wang Q, Navitskaya S, Chakravarthy H, Huang C, Kady N, Lydic TA, et al. Dual anti-inflammatory and anti-angiogenic action of miR-15a in diabetic retinopathy. EBioMedicine (2016) 11:138-50. doi:10.1016/j. ebiom.2016.08.013

104. Zhou Q, Frost RJ, Anderson C, Zhao F, Ma J, Yu B, et al. Let-7 contributes to diabetic retinopathy but represses pathological ocular angiogenesis. Mol Cell Biol (2017) 37:e00001-17. doi:10.1128/MCB.00001-17

105. Ding Y, Sun X, Shan P-F. MicroRNAs and cardiovascular disease in diabetes mellitus. Biomed Res Int (2017) 2014:4080364. doi:10.1155/2017/4080364

106. Kong L, Zhu J, Han W, Jiang X, Xu M, Zhao Y, et al. Significance of serum microRNAs in pre-diabetes and newly diagnosed type 2 diabetes: a clinical study. Acta Diabetol (2011) 48:61-9. doi:10.1007/s00592-010-0226-0

107. Shantikumar S, Caporali A, Emanueli C. Role of miRNA in diabetes and its cardiovascular complications. Cardiovasc Res (2012) 93(4):583-93. doi:10.1093/cvr/cvr300

108. Qing S, Yuan S, Yun C, Hui H, Mao P, Wen F, et al. Serum miRNA biomarkers serve as a fingerprint for proliferative diabetic retinopathy. Cell Physiol Biochem (2014) 34:1733-40. doi:10.1159/000366374

109. Barutta F, Bruno G, Matullo G, Chaturvedi N, Grimaldi S, Schalkwijk C, et al. MicroRNA-126 and micro-/macrovascular complications of type 1 diabetes in the EURODIAB prospective complications study. Acta Diabetol (2017) 54:133-9. doi:10.1007/s00592-016-0915-4

110. Umeda PK, Shiau RP, Miggins M, Caufield JB. Epigenetic alterations in diabetic cardiomyopathy. J Mol Cell Cardiol (2001) 33:A124. doi:10.1016/ S0022-2828(01)90494-8

111. Mohan A, Singh RS, Kumari M, Garg D, Upadhyay A, Ecelbarger CM, et al. Urinary exosomal microRNA-451-5p is a potential early biomarker of diabetic nephropathy in rats. PLoS One (2016) 11:e0154055. doi:10.1371/ journal.pone. 0154055
112. Meng S, Cao J, Zhang X, Fan Y, Fang L, Wang C, et al. Downregulation of microRNA-130a contributes to endothelial progenitor cell dysfunction in diabetic patients via its target Runx3. PLoS One (2013) 8:e68611. doi:10.1371/ journal.pone.0068611

113. Ruiz MA, Feng B, Chakrabarti S. Polycomb repressive complex 2 regulates miR-200b in retinal endothelial cells: potential relevance in diabetic retinopathy. PLoS One (2015) 10:e0123987. doi:10.1371/journal.pone.0123987

114. Wang Y, Yan H. MicroRNA-126 contributes to Niaspan treatment induced vascular restoration after diabetic retinopathy. Sci Rep (2016) 6:26909. doi:10.1038/srep26909

115. Hou Q, Zhou L, Tang J, Ma N, Xu A, Tang J, et al. LGR4 is a direct target of microRNA-34a and modulates the proliferation and migration of retinal pigment epithelial ARPE-19 cells. PLoS One (2016) 11:e0168320. doi:10.1371/ journal.pone.0168320

116. Zampetaki A, Kiechl S, Drozdov I, Willeit P, Mayr U, Prokopi M, et al. Plasma microRNA profiling reveals loss of endothelial miR-126 and other microRNAs in type 2 diabetes. Circ Res (2010) 107:810-7. doi:10.1161/ CIRCRESAHA.110.226357

117. Zhang M, Gu H, Xu W, Zhou X. Down-regulation of IncRNA MALAT1 reduces cardiomyocyte apoptosis and improves left ventricular function in diabetic rats. Int J Cardiol (2016) 203:214-6. doi:10.1016/j.ijcard.2015.10.136

118. Moran VA, Perera RJ, Khalil AM. Emerging functional and mechanistic paradigms of mammalian long non-coding RNAs. Nucleic Acids Res (2012) 40:6391-400. doi:10.1093/nar/gks296

119. Zhuo C, Jiang R, Lin X, Shao M. IncRNA H19 inhibits autophagy by epigenetically silencing of DIRAS3 in diabetic cardiomyopathy. Oncotarget (2017) 8:1429. doi:10.18632/oncotarget.13637

120. Wang S, Xu H, Zou L, Xie J, Wu H, Wu B, et al. IncRNA uc. 48+ is involved in diabetic neuropathic pain mediated by the $\mathrm{P} 2 \mathrm{X} 3$ receptor in the dorsal root ganglia. Purinergic Signal (2016) 12:139-48. doi:10.1007/s11302-015-9488-x

121. Cesana M, Cacchiarelli D, Legnini I, Santini T, Sthandier O, Chinappi M, et al. A long noncoding RNA controls muscle differentiation by functioning as a competing endogenous RNA. Cell (2011) 147:358-69. doi:10.1016/j. cell.2011.09.028

122. Hu M, Wang R, Li X, Fan M, Lin J, Zhen J, et al. IncRNA MALAT1 is dysregulated in diabetic nephropathy and involved in high glucose-induced podocyte injury via its interplay with $\beta$-catenin. J Cell Mol Med (2017). Available from: http://onlinelibrary.wiley.com/doi/10.1111/jcmm.13189/full

123. Zhou L, Xu DY, Sha WG, Shen L, Lu GY, Yin X. Long non-coding MIAT mediates high glucose-induced renal tubular epithelial injury. Biochem Biophys Res Commun (2015) 468:726-32. doi:10.1016/j.bbrc.2015.11.023

124. Shan K, Li C-P, Liu C, Liu X, Yan B. RNCR3: a regulator of diabetes mellitus-related retinal microvascular dysfunction. Biochem Biophys Res Commun (2017) 482:777-83. doi:10.1016/j.bbrc.2016.11.110

Conflict of Interest Statement: The authors declare that the research was conducted in the absence of any commercial or financial relationships that could be construed as a potential conflict of interest.

Copyright (C) 2017 Khullar, Cheema and Raut. This is an open-access article distributed under the terms of the Creative Commons Attribution License (CC BY). The use, distribution or reproduction in other forums is permitted, provided the original author(s) or licensor are credited and that the original publication in this journal is cited, in accordance with accepted academic practice. No use, distribution or reproduction is permitted which does not comply with these terms. 